Musées, Patrimoine et Culture scientifiques et techniques

$160 \mid 2015$

juillet-août 2015

\title{
Exposer les découvertes archéologiques des grands tracés
}

\section{Astrid Chevolet}

\section{OpenEdition}

\section{Journals}

Édition électronique

URL : http://journals.openedition.org/ocim/1544

DOI : 10.4000/ocim.1544

ISSN : 2108-646X

Éditeur

OCIM

Édition imprimée

Date de publication : 1 juillet 2015

Pagination : 17-21

ISSN : 0994-1908

Référence électronique

Astrid Chevolet, «Exposer les découvertes archéologiques des grands tracés », La Lettre de l'OCIM [En ligne], 160 | 2015, mis en ligne le 01 juillet 2016, consulté le 14 novembre 2019. URL : http:// journals.openedition.org/ocim/1544

Ce document a été généré automatiquement le 14 novembre 2019.

Tous droits réservés 


\title{
Exposer les découvertes archéologiques des grands tracés
}

\author{
Astrid Chevolet
}

L'entrée de l'exposition C'était là ! Sous nos pieds...

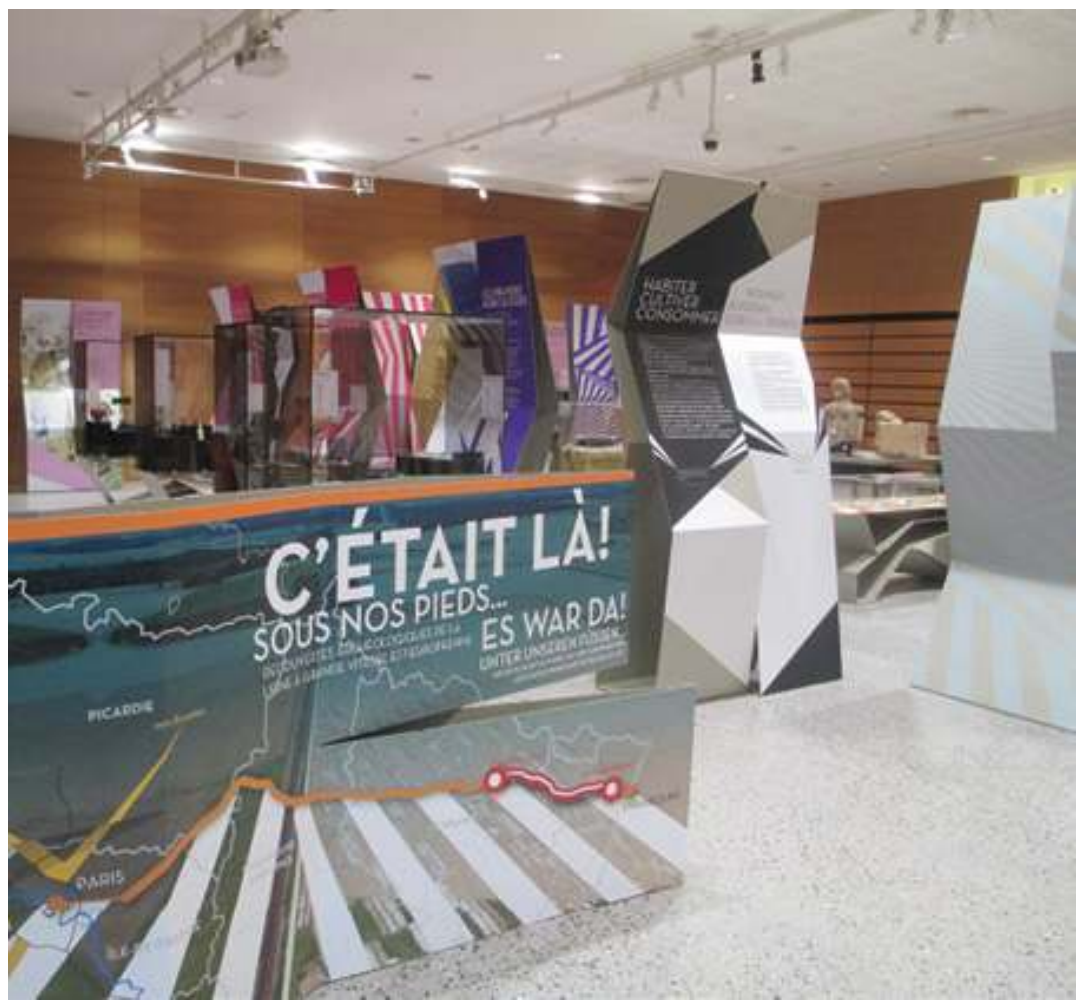

(c) Inrap/Astrid Chevolet

Les expositions consacrées aux opérations d'archéologie préventive sur de grands tracés présentent plusieurs spécificités liées au nombre important de données et d'objets à traiter pour une valorisation intelligible par le public. 
2 L'aménagement de la Ligne à Grande Vitesse (LGV) Est européenne entre Paris et Strasbourg a donné lieu à la réalisation de deux expositions itinérantes, Cent mille ans sous les rails (accueillie à Nancy, Soissons et au musée d'Archéologie nationale à SaintGermain-en-Laye de 2006 à 2010) et C'était là ! Sous nos pieds... / Es war da! Unter unseren Füssen... correspondant aux deux phases du tracé. L'importance du nombre de données à traiter, la proximité temporelle de l'exposition et des fouilles archéologiques, le traitement particulier du mobilier issu de fouilles archéologiques préventives, sont des éléments caractéristiques de ce type de projet. C'était là ! Sous nos pieds... est le fruit d'un partenariat entre l'Institut national de recherches archéologiques préventives (Inrap, établissement public créé en 2001 en application du code du Patrimoine - Livre V, Titre II, loi sur l'archéologie préventive du 17 janvier 2001 - et placé sous double tutelle des ministères de la Culture et de la Communication et de l'Enseignement supérieur et de la Recherche), également maîtrise d'ouvrage déléguée, le ministère de la Culture et de la Communication, Réseau ferré de France et le Pôle d'archéologie interdépartemental rhénan (PAIR). L'Inrap est intervenu en amont de l'aménagement de la LGV Est européenne, qui s'est déroulé en deux phases. La première phase couvrait $300 \mathrm{~km}$ et traversait quatre régions, l'île-de-France, le Nord-Pas de Calais, la Champagne et la Lorraine. Les découvertes ont été présentées dans le cadre de l'exposition itinérante Cent mille ans sous les rails, en partenariat avec le ministère de la Culture et de la Communication, l'Inrap et Réseau ferré de France. La deuxième phase de la LGV Est, d'une longueur plus modeste de $106 \mathrm{~km}$, a donné lieu à l'exposition C'était là ! Sous nos pieds... conçue elle aussi comme itinérante puisqu'aucun des partenaires ne disposait d'un lieu d'accueil. Le mobilier muséographique de Cent mille ans sous les rails, composé de grandes cimaises et vitrines métalliques, a été réemployé ${ }^{1}$. La cible de ces deux expositions était un public régional et familial, l'enjeu pour les acteurs du projet étant de s'adresser en priorité aux visiteurs impactés par les travaux de la ligne.

\section{Les fouilles archéologiques de la Ligne à Grande Vitesse Est européenne}

3 Depuis 2001, la procédure d'archéologie préventive implique, sur prescription des services de l'État (Directions régionales des Affaires culturelles), la réalisation de diagnostics archéologiques puis de fouilles si ces derniers se révèlent positifs. Un diagnostic est une méthode de détection basée sur des tranchées réalisées à l'aide d'engins mécaniques. En creusant jusqu'au niveau d'apparition des vestiges, les archéologues déterminent si des structures sont présentes sous la surface. D'autres méthodes de détection, exploitées en complément, fournissent des données sur le soussol : prospections aériennes, pédestres et géophysiques, carottages ou encore sondages géotechniques. Le LIDAR (Light detection and ranging), scanner laser aéroporté exploité par Réseau ferré de France, a également été très utile aux archéologues lors des opérations menées sur la seconde phase, fournissant de précieuses données sur les zones boisées.

Cette procédure a été mise en œuvre sur l'ensemble du tracé de la LGV Est européenne. Les opérations menées sur la première phase entre 2000 et 2004 ont concerné $300 \mathrm{~km}$ de tracé, donnant lieu à la découverte de 171 sites d'habitat et de 20 nécropoles. La deuxième phase était plus modeste, avec 40 sites découverts. La Ligne à Grande Vitesse traverse des territoires ruraux, souvent peu documentés du point de vue 
archéologique. Diagnostics et fouilles ont mis en évidence des peuplements et une exploitation très ancienne des sols. Ils ont également mis au jour un nombre important de vestiges archéologiques. L'enjeu des deux expositions, Cent mille ans sous les rails et C'était là ! Sous nos pieds... était de parvenir à valoriser les découvertes scientifiques faites par les archéologues en se servant des objets comme témoins, comme relais vers les notions qu'ils illustrent.

Vue aérienne du site de Duntzenheim (Haut-Rhin) après son décapage.

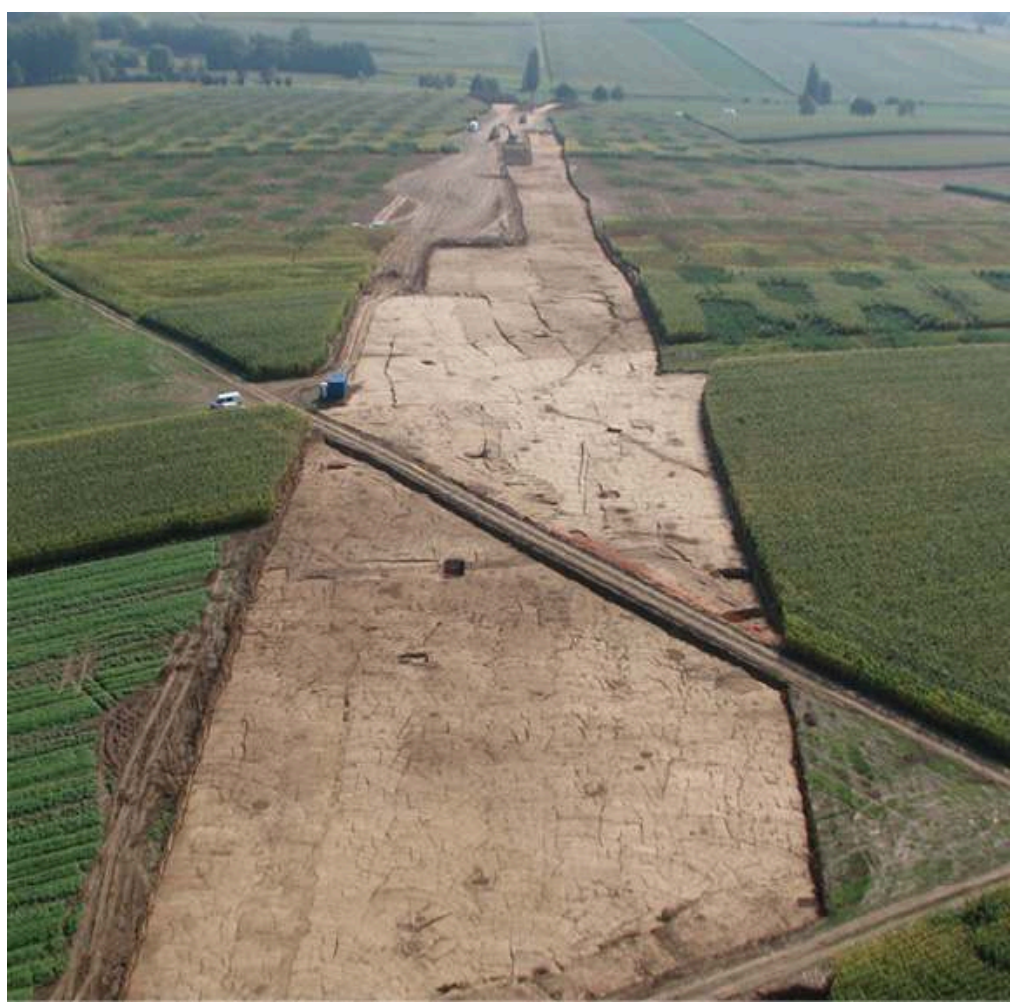

(C) Inrap/2C2L

\section{Le scénario et le parcours : une matière dense à organiser}

5 Les fouilles archéologiques menées sous la LGV Est ne présentent parfois qu'un seul lien entre elles : être situées sur le tracé. La matière à traiter était donc non seulement très dense, allant du Néolithique à la Première Guerre mondiale, des sites funéraires aux sites d'habitat, l'artisanat, les sites cultuels, mais le fil conducteur entre ces données n'était pas toujours facile à déterminer. Les résultats tiennent à la physionomie du tracé lui-même: des sites ruraux uniquement, puisque la LGV n'a pas vocation à traverser des villes, des sites agricoles essentiellement, des villages peu ou pas documentés du point de vue archéologique... La répartition des périodes elle-même est différente selon les régions: la Moselle, par exemple, fait la part belle aux sites romains, tandis que les sites à proximité de Strasbourg remontent au Néolithique ancien. Peu de sites modernes pour la seconde phase, ce qui provoque un long hiatus entre les découvertes médiévales et la tombe des deux poilus de la Première Guerre mondiale (Sarraltroff, Moselle). Puisque l'objectif était de faire état des découvertes 
réalisées sur le tracé, il était difficile de s'intéresser à un site en particulier en développant de manière trop détaillée le contexte archéologique de ce dernier. Le scénario et le parcours ont demandé un important travail de collecte des données, de synthèse et de mise en perspective, afin de dégager une cohérence. Parmi les 40 sites fouillés sur la seconde phase, une dizaine se distinguent, soit par la richesse du mobilier, soit par le caractère exceptionnel de la découverte. Ce sont essentiellement ces derniers qui sont présentés dans l'exposition C'était là! Sous nos pieds..., même si le parcours permet d'évoquer d'autres découvertes, plus modestes mais néanmoins significatives.

Fouille d'une sépulture sur le site d'Ingenheim (Bas-Rhin).

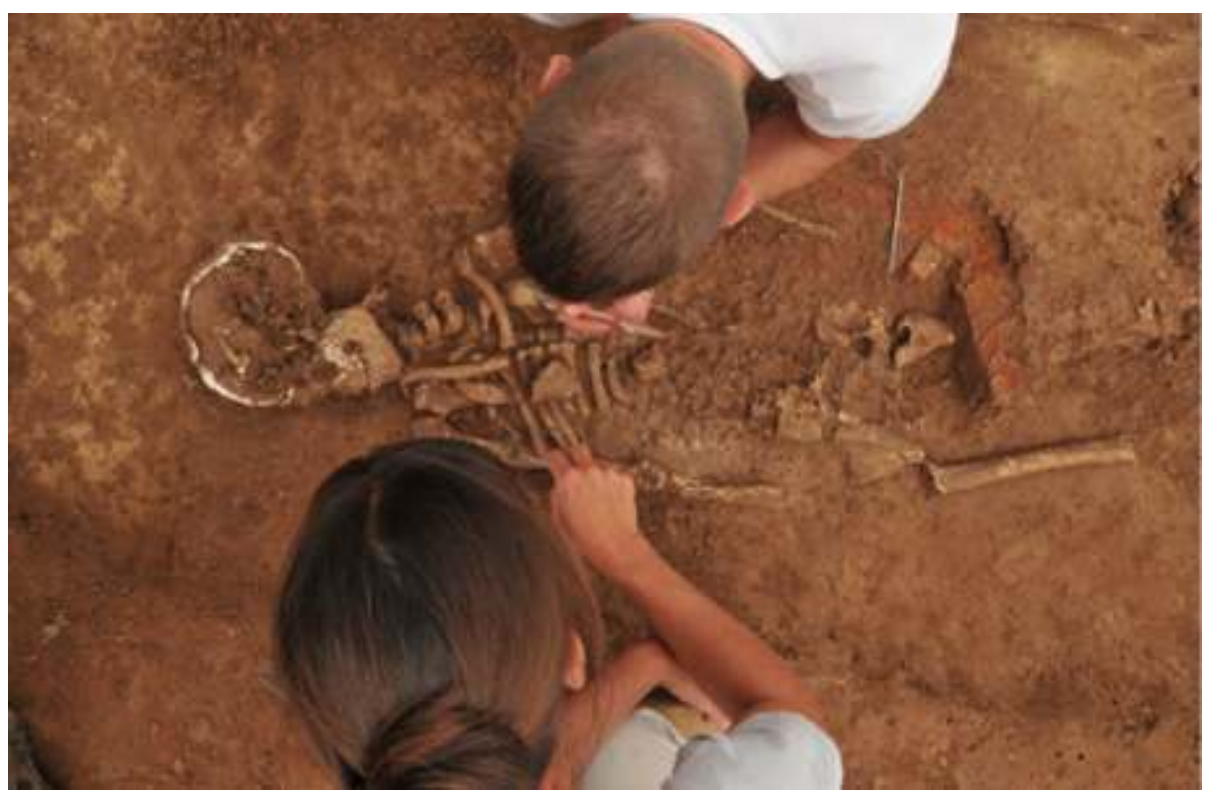

(c) Inrap/François Schneikert

6 Comme souvent, l'un des choix les plus difficiles était le suivant: découpage chronologique ou thématique? Cent mille ans sous les rails avait fait le choix d'un découpage thématique, imaginé comme un zoom allant du territoire dans sa notion la plus large ("Des Paysages en mouvement") à ses dimensions les plus intimes ("Le territoire des morts"). Dans le processus de réflexion du commissariat scientifique de l'exposition C'était là! Sous nos pieds... un découpage chronologique a tout d'abord été envisagé. Mais les mêmes questions sont revenues : comment mettre en évidence les notions accrochant l'intérêt du visiteur, comme souligner les processus diachroniques, comment éviter les coupures artificielles alors que les résultats des opérations montraient, pour plusieurs problématiques, une continuité ? Les opérations de la LGV Est témoignaient dans certains cas d'une pérennité des pratiques d'une période à l'autre, comme la coutume de l'inhumation en silo, apparue au Néolithique et qui persiste encore à la période gauloise. Dégager des problématiques évocatrices pour le visiteur, comme l'habitat, les modes de cultures, les rites funéraires, les mettre en relation au gré des périodes, paraissait plus intéressant; encore fallait-il disposer de ces exemples pour toutes les périodes, ce qui était loin d'être le cas. C'est donc une solution intermédiaire qui a été privilégiée : un parcours thématique, mais à l'intérieur duquel la chronologie est également présente. Les trois thématiques sont les suivantes : "Habiter, cultiver, consommer", "Fabriquer, échanger, se déplacer" et "Croire, mourir, 
se souvenir". Chaque thématique débute par la présentation d'un site archéologique, envisagé sous l'angle de celle-ci. Ce premier site diachronique est suivi de cimaises et vitrines organisées par ordre chronologique et mettant en évidence une découverte spécifique d'une période. Par exemple, "Habiter, cultiver, consommer" présente successivement les premiers agriculteurs du Néolithique, l'alimentation des Gaulois, les villae romaines (résidence et bâtiments agricoles), La bergerie médiévale de Lucy (Moselle). Tout comme Cent mille ans sous les rails, C'était là! Sous nos pieds... se clôt sur la thématique consacrée aux sites funéraires, "Croire, mourir, se souvenir". La contrainte était de limiter les textes à 500 signes, tant pour la compréhension du lecteur que pour des problèmes de places, les données textuelles étant systématiquement traduites en allemand.

La situle, seau en Bronze d'influence étrusque, découverte dans une tombe de l'Âge du Fer à Eckwersheim (Bas-Rhin), mise à jour en 2010, est l'une des pièces les plus importantes de l'exposition.

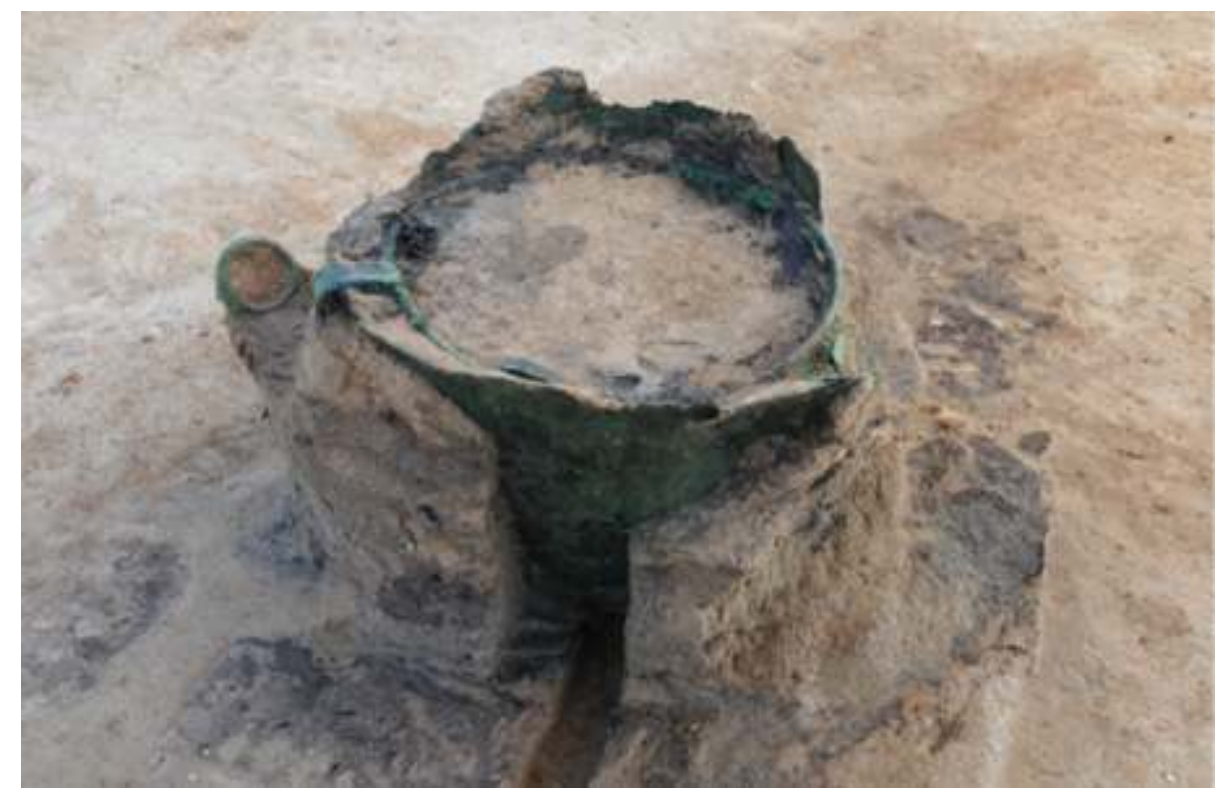

(C) Inrap

La matérialisation du parcours dans l'espace présente plusieurs faiblesses. Les repères chronologiques ont été restitués par la scénographie, mais le principe adopté ne donne pas entièrement satisfaction. En effet les codes couleurs ne sont pas rappelés sur chaque cimaise, de sorte qu'il est difficile de se souvenir, devant une cimaise saumon, que cette couleur désigne la période médiévale. Si le texte des panneaux reprend systématiquement les indications chronologiques, une seule mention ne suffit pas et complique la visite. Les différentes thématiques sont également peu soulignées; chacune est présentée par une cimaise introductive, mais le découpage est peu sensible dans le parcours, tout comme le sens de la visite. L'équipe du Parc archéologique de Bliesbruck a ajouté au bout de quelques jours un panneau au début du parcours, les visiteurs ayant tendance à se diriger directement vers la fin de l'exposition. 
"Habiter, Cultiver, Consommer" : la première séquence de C\&apos ;était là ! Sous nos pieds...

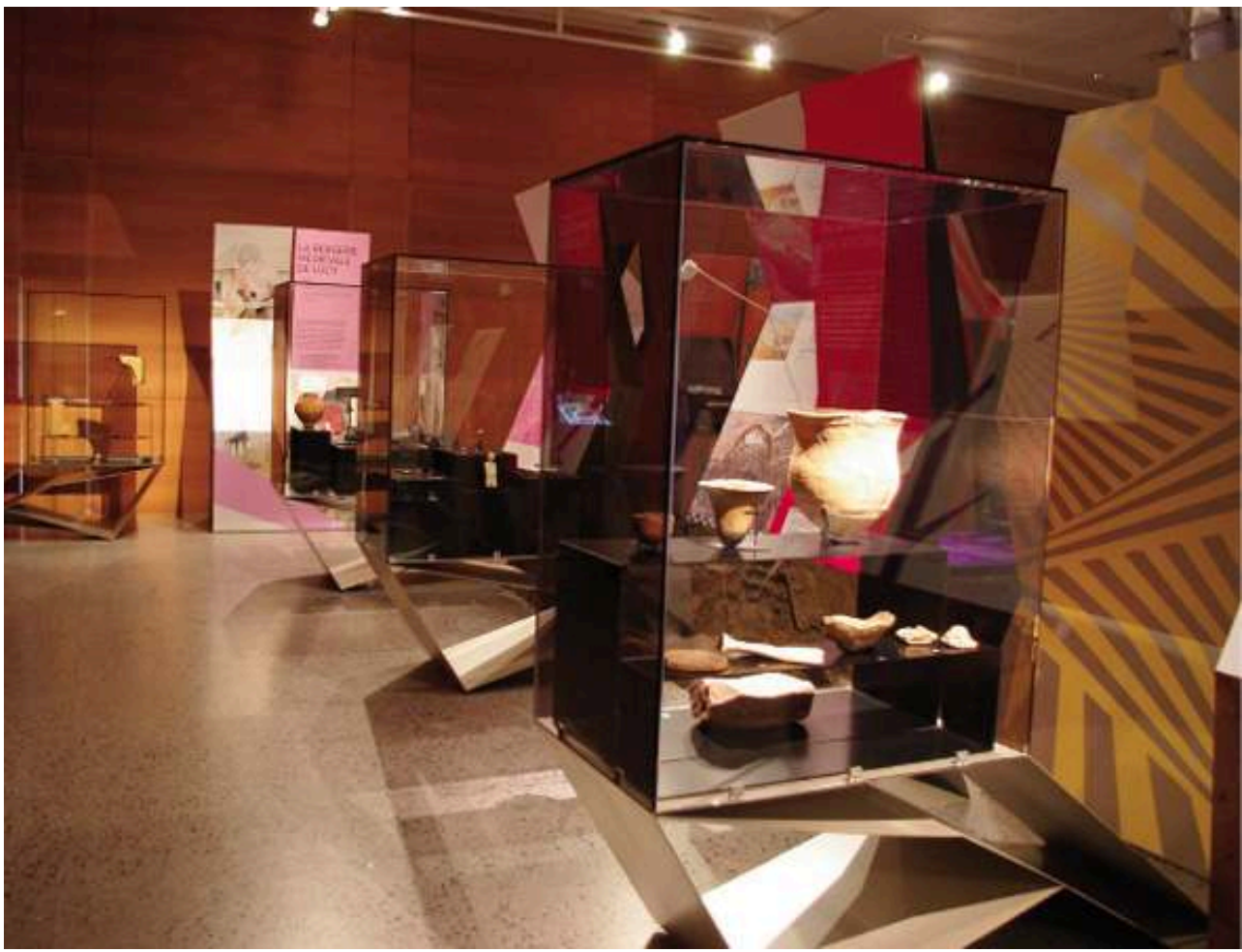

(C) Inrap/Astrid Chevolet

\section{Le mobilier archéologique}

Réaliser une sélection d'objets issus de fouilles préventives récentes présente plusieurs difficultés. Le nombre d'objets d'abord: des milliers d'artefacts ont été découverts dans le cadre des fouilles archéologiques de la LGV Est. Le choix a été fait d'en présenter un nombre conséquent, presque 350 objets pour la seconde phase, en comptant les pièces issues du dépôt monétaire gaulois de Bassing (Moselle). Ces objets vont du tesson de poterie à la bague en métal précieux en passant par le mobilier lapidaire, les fragments de meule, les minuscules perles en verre... Les responsables des opérations archéologiques, les gestionnaires de collection et le commissariat scientifique ont contribué à une première sélection. Certaines pièces ont été ensuite exclues, du fait de leur fragilité. Un second lot a été confié à différents laboratoires pour leur restauration. Il s'agissait de deux types d'objets: les objets en métal (fer, bronze) et certaines céramiques. L'essentiel des objets en métal a été traité par le LAM (Laboratoire d'archéologie des métaux, Nancy-Jarville), la céramique par le CREAM (Centre de restauration et d'étude archéologique municipal, Vienne). La stabilisation du mobilier ferreux, destinée à éviter la reprise de la corrosion, a nécessité plusieurs mois. Un objet a bénéficié d'une prise en charge spécifique : une situle, seau en bronze d'importation étrusque daté de 800 avant notre ère et mis au jour dans une tombe de l'Âge du Fer à Eckwersheim (Bas-Rhin). Sa restauration, spectaculaire, a été réalisée à Mayence en Allemagne, par le Römisch-Germanisches Zentralmuseum (RGZM).

9 Le règlement du statut des objets dans le respect de la législation a également demandé une solide collaboration entre les différents acteurs. Un objet découvert dans le cadre 
d'une fouille archéologique préventive appartient pour moitié à l'État, pour moitié au propriétaire de la parcelle au moment de l'opération. Selon le Code du Patrimoine (article L 523-14), "La propriété du mobilier archéologique issu des opérations d\&apos;archéologie préventive est partagée à parts égales entre l\&apos, État et le propriétaire du terrain. Si, à I\&apos ;issue d\&apos ;un délai d\&apos ;un an à compter de la réception du rapport de fouilles mentionné à $1 \& a p o s$;article L. 523-11, le propriétaire n\&apos;a pas exprimé une intention contraire, il est réputé avoir renoncé à la propriété des vestiges qui lui étaient échus par le partage. La propriété de ces vestiges est alors transférée à titre gratuit à l\&apos ;État (...)". Le statut du mobilier archéologique devrait être revu dans le cadre de la réforme du Code $d u$ Patrimoine envisagée par le ministère de la Culture et de la Communication.

L'une des céramiques les plus importantes est une jarre funéraire de l'Âge du Bronze. Elle a été confiée au CREAM pour sa consolidation, ici réalisée par Marylen Kappes (restauratrice verre et céramique).

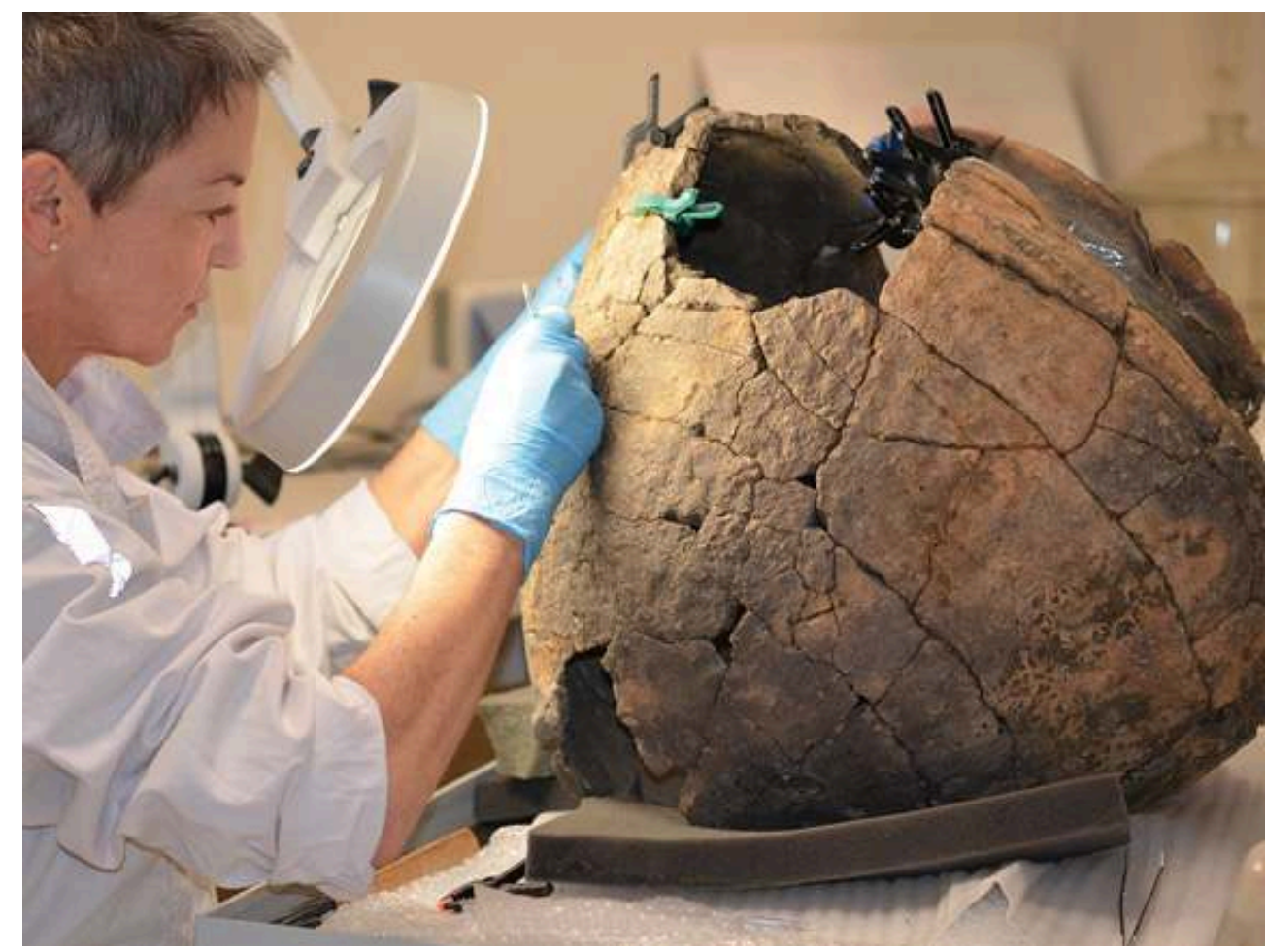

(c) CREAM Vienne

10 Cependant Réseau ferré de France n'était pas toujours propriétaire des parcelles lorsque les archéologues sont intervenus. De plus, quelques fouilles couvraient plusieurs parcelles, ayant pour certaines chacune un propriétaire différent... Une fois la dévolution du mobilier réalisée par l'État, le statut de ces objets sera clarifié et ils seront déposés dans les différents musées des régions concernées ou encore dans les centres de conservation et d'étude régionaux. Ce long processus n'avait pas encore abouti lors de la conception de l'exposition C'était là! Sous nos pieds.... Les services régionaux de l'archéologie de Lorraine et d'Alsace devaient donc solliciter l'accord préalable des propriétaires des parcelles afin que la présentation du mobilier se fasse en conformité avec la législation.

Le stockage d'un nombre aussi important d'objets, sans parler des prélèvements, pose la question de la place disponible dans les réserves des différents musées, à laquelle 
doivent répondre les Centres de conservation et d'étude (CCE) gérés par les services régionaux de l'archéologie, dont certains sont encore en construction. Quoiqu'il en soit, les objets étant alors répartis selon leur région d'origine, les expositions consacrées aux grands linéaires sont le seul moment où les objets issus du tracé peuvent être présentés ensemble, permettant au public d'appréhender l'intérêt des opérations d'archéologie préventive sur des tracés de grands linéaires.

\section{Conclusion}

La réalisation C'était là! Sous nos pieds... a sans aucun doute bénéficié de l'expérience de l'exposition précédente, Cent mille ans sous les rails, permettant d'anticiper plusieurs aspects lors du déroulement des fouilles archéologiques de la seconde phase du tracé. Une exposition nécessite des prises de vues différentes de celles réalisées par les archéologues. Cette difficulté s'étant posée lors de la conception de Cent mille ans sous les rails, des campagnes de prise de vues ont pu être programmées. Moins riche en sites, C'était là! Sous nos pieds... présente davantage de mobilier que Cent mille ans sous les rails, la sélection et les restaurations du mobilier ayant pu être mieux intégrées au processus de conception. Le découpage du scénario s'en inspire également. Plus courts que ceux de Cent mille ans sous les rails, les textes de C'était là! Sous nos pieds... sont également bilingues français/allemand. Ce choix est fortement lié à l'accueil de l'exposition au Parc archéologique européen de Bliesbruck. Ces deux expositions témoignent enfin de la nécessité d'évaluer la faisabilité de projets de ce type dans le cadre d'opérations d'archéologie préventive, et ce bien en amont. Les spécificités des lieux d'exposition doivent en outre être prises en compte dès l'origine, sous peine de se retrouver avec une exposition trop peu modulable et difficile à proposer.

C'était là! Sous nos pieds... a été présentée au Parc archéologique européen de Bliesbruck - Reinheim du 16 mai au 30 septembre 2014. 15862 personnes ont visité le Parc entre le 16 mai et le 8 septembre et au moins 7904 visiteurs allemands, soit près de 24000 personnes ${ }^{2}$. Le musée du Pays de Sarrebourg l'accueille jusqu'au 31 août 2015. Elle devrait ensuite être présentée à Strasbourg ou encore à Metz. Les retours des visiteurs sont essentiellement positifs et concernent les objets ainsi que le caractère pédagogique de la présentation. Plusieurs visiteurs déplorent néanmoins le manque d'éclairage, le caractère succinct des cartels des vitrines ou encore la hauteur de cellesci. Certains ont également ressenti une absence de cohérence dans le parcours. Ces retours incitent à bien recentrer ses objectifs lorsque l'on conçoit une exposition de ce type. Si l'accent doit être mis sur l'objet, une attention constante doit être portée à la clarté et à la lisibilité des cartels tout comme aux conditions d'exposition. En conséquence, le parcours doit être le plus lisible possible et ne pas écarter, au final, une présentation par ordre chronologique, plus intuitive. 


\section{NOTES}

1. La scénographie de ces deux expositions a été confiée à l'agence Zen+Dco.

2. Données transmises par Alexandra Vincent, responsable des publics du Parc archéologique européen de Bliesbruck, le 9 septembre 2014. Le questionnaire de satisfaction a été complété par 52 visiteurs et contient $79 \%$ de retour type «très satisfaisant ». Les remarques sont issues à la fois de l'enquête et du Livre d'or.

\section{RÉSUMÉS}

Permettre à un public le plus large possible d'accéder aux résultats de découvertes issues de fouilles archéologiques préventives : tel était le principal objectif d'une exposition itinérante dont l'une des responsables présente ici les contraintes et les partenariats ainsi que les choix finalement effectués.

\section{INDEX}

Mots-clés : fouille archéologique, archéologie préventive

\section{AUTEUR}

\section{ASTRID CHEVOLET}

responsable de la coordination des actions de culture scientifique au Jardin des sciences de l'université de Strasbourg et auparavant chef de projet d'exposition à l'Institut national de recherches archéologiques préventives

astrid.chevolet@unistra.fr 